PEB Échanges, Programme pour la construction et l'équipement de l'éducation 2005/08

\title{
Un système de gestion des actifs immobiliers au Québec
} Dino Gerbasi 
UN SYSTÈME DE GESTION DES ACTIFS IMMOBILIERS AU QUÉBEC

Au cours des dernières années les services des ressources matérielles des commissions scolaires québécoises ont été confrontés à plusieurs problèmes liés à la gestion de leur parc immobilier. Premièrement, le vieillissement des immeubles qui ont majoritairement été construits durant les années 50 à 70 fait en sorte que de nombreux composants, ayant atteint depuis peu la fin de leur vie utile, requièrent des réfections majeures (voir figure 1). Par ailleurs, les ressources financières s'avèrent limitées puisqu'elles sont ajustées uniquement en partie pour tenir compte des besoins liés à l'âge du parc. Enfin, le départ à la retraite $d^{\prime}$ un très grand nombre d'employés expérimentés ou de gestionnaires met en évidence la fragilité de l'expertise et de la connaissance relatives à l'état des immeubles.

\section{Figure 1}

Parc immobilier des commissions scolaires du Québec : ajouts de superficie construite - par période de dix ans

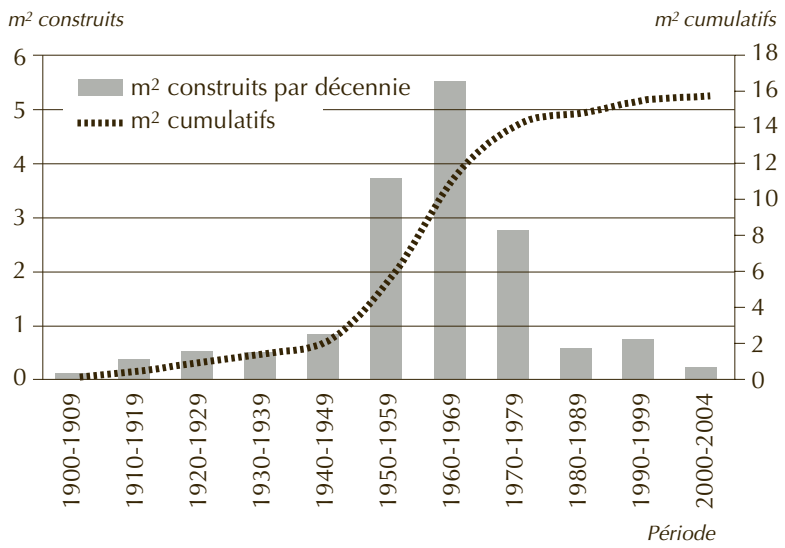

Ces problèmes rendent difficile, pour plusieurs commissions scolaires, la séléction annuelle optimale des nombreux travaux de réfection à réaliser, en tenant compte d'une vision à long terme du service à offrir.

En concertation entre les commissions scolaires et le ministère de l'Éducation, il a donc été convenu de rendre disponible, pour l'usage du personnel des ressources matérielles des commissions scolaires, de leurs gestionnaires et de ceux du ministère de l'Éducation, un outil informatique de dernière génération pour aider au maintien des actifs immobiliers. Un logiciel ne peut bien sûr solutionner un éventuel manque de ressources financières consacrées aux réparations majeures mais à tout le moins assure-t-il que les ressources, lorsqu'elles sont rendues disponibles, sont mieux dépensées.
Nous présenterons ici les principales raisons justifiant un système de gestion des actifs immobiliers (SGA), un bref historique de leur évolution et la démarche entreprise par le Québec visant à mettre en place un tel système. Les principales caractéristiques recommandées d'un système de gestion des actifs se trouvent en annexe.

\section{Pourquoi un SGA}

Un système de gestion des actifs immobiliers efficace permet aux gestionnaires de bien identifier les activités de maintien d'actifs et de dresser un portrait précis pour les décideurs quant à l'état des actifs immobiliers et aux scénarios d'investissement à envisager. Avec un tel outil, les recommandations des gestionnaires sont alors bien supportées et peuvent traverser plus facilement le processus décisionnel avec succès.

Pourquoi se doter d'un tel outil maintenant? Contrairement aux années antérieures qui étaient orientées vers la conception-construction, les prochaines années seront consacrées aux réparations et aux rénovations. Les gestionnaires immobiliers d'aujourd'hui font face à des décisions complexes relatives à la sélection, à la modification et au remplacement d'actifs immobiliers. Voici quelques facteurs qui peuvent influencer leurs décisions :

- Le changement de mission ou de vocation d'un immeuble.

- Les changements environnementaux.

- L'opportunité d'optimiser la capacité d'un actif lors de son remplacement en fin de la vie utile.

- Les impacts causés par les fluctuations des coûts énergétiques et la disponibilité de nombreuses sources de combustible sur la sélection d'une technologie de chauffage et de climatisation d'un bâtiment.

- Les changements démographiques.

Un SGA fournit aux gestionnaires des données importantes relatives à l'évolution de leurs actifs immobiliers et leur permet de prendre des décisions éclairées, basées sur les prévisions de dépenses d'investissement et de fonctionnement prenant en compte tout le cycle de vie utile de l'actif. À titre d'exemple le SGA facilitera la prise de décision lors du remplacement d'équipements de chauffage ou de climatisation car il intégrera les renseignements sur les coûts d'acquisition, d'installation et d'entretien de chaque équipement pouvant être retenu, et prendra également en considération sa durée de vie utile et sa performance énergétique.

\section{Évolution des SGA}

Contrairement aux systèmes de maintenance assistée par ordinateur (GMAO) qui offrent des fonctionnalités généralement uniformisées et des méthodes et pratiques qui ont été améliorées au fil des ans, les systèmes de gestion des actifs immobiliers qui doivent répondre à un nouveau marché n'offrent pas encore de caractéristiques 
et approches constantes. Cette situation est également amplifiée par le fait que certains GMAO sont proposés à titre de système de gestion des actifs car ils incorporent la notion de durée de vie.

Les premières générations de systèmes de gestion des actifs immobiliers telles Dataquire, BUILDER et PEB sont apparues sur le marché au milieu des années 1990. Ces dernières incorporaient les calculs de coûts sur le cycle de vie des principaux types d'actifs immobiliers utilisant des coûts de cycle de vie prédéfinis. Ces outils novateurs projetaient des coûts de maintien et de réhabilitation stratégique des actifs sur une période de 50 ans. Ils étaient utilisés principalement à titre de références et d'outils d'analyse de performance. Cependant, des lacunes au niveau de leur capacité à gérer les inventaires d'actifs imposants étaient observées.

Comme les systèmes de maintenance assistée par ordinateur possédaient ces deux fonctionnalités, ceci ouvrait la porte aux développeurs pour en faire des outils potentiels de maintien des actifs immobiliers. Toutefois, ils ont vite réalisé que la tâche était beaucoup plus complexe que de simplement ajouter quelques champs paramétrables dans le module d'inventaire des actifs.

Ce changement nécessitait l'intégration des principes de calcul des coûts de cycle de vie, une connaissance complète des bâtiments et une base de données exhaustive et évolutive des coûts de cycle de vie. Au lieu d'investir dans ce champ d'activité et diversifier leurs activités, la majorité des développeurs de systèmes de maintenance assistée par ordinateur ont décidé de s'en tenir à leurs activités principales. À partir de ce moment, nous avons assisté à une spécialisation des fournisseurs de SGA et des fournisseurs de GMAO.

Nous avons vu apparaître par la suite la nouvelle génération de systèmes de gestion des actifs immobiliers tels SARRA $^{1}$, RECAPP ${ }^{2}$, VFA. facility ${ }^{3}$, et Antilope ${ }^{4}$. Bien que leurs approches soient diversifiées, ces produits incluent tous un module de prévision des coûts de cycle de vie, des fonctionnalités de planification des travaux et un module détaillé pour gérer l'état des équipements.

Aujourd'hui, dans nos organisations, la recherche de bases de données référentielles et l'évolution rapide des technologies de l'information suggèrent la mise en lien des systèmes de gestion des actifs et des systèmes de maintenance assistée par ordinateur. Comme les GMAO sont considérés par beaucoup comme le point d'entrée des données relatives aux actifs, I'une des tendances du marché actuel consis-

1. SARRA (Système d'Allocation des Ressources et de Réhabilitation des Actifs) de GES Technologies.

2. RECAPP de Physical Planning Technologies.

3. VFA.facility de VFA.

4. Antilope de Socotec. tera à développer des interfaces entre les deux outils : le système de gestion des actifs immobiliers et le système de maintenance assistée par ordinateur.

\section{La mise en place d'un SGA}

Voici les étapes qui sont présentement suivies par les commissions scolaires du Québec et le ministère de I'Éducation et qui conduiront, vraisemblablement en 2005, à la mise en place d'un système de gestion des actifs immobiliers adapté aux besoins des utilisateurs :

1. Former un groupe de travail conjoint pour s'assurer que les réalités et besoins des commissions scolaires, de même que ceux du ministère, soient pris en compte.

2. Identifier des besoins de base et des caractéristiques du système, tels que :

- Simple d'utilisation.

- Utilisé par toutes les commissions scolaires.

- Utile au personnel de tous les niveaux impliqués dans la gestion du parc, aussi bien dans les commissions scolaires qu'au ministère, prenant pour acquis que les commissions scolaires sont responsables des décisions de maintien des actifs et le ministère des orientations et du financement.

- Accessible sur Internet.

- Développable pour permettre, dans le futur, l'ajout de fonctions et une mise en lien avec d'autres logiciels.

3. Retenir une orientation : il fut décidé d'acheter les droits d'utilisation d'un système existant et de le faire modifier légèrement pour l'adapter aux réalités et besoins spécifiques du réseau.

4. Rencontrer des firmes offrant ce type de système.

5. Recevoir l'approbation des autorités de passer à la phase de mise en œuvre du SGA.

6. Accepter la proposition d'une firme; le Québec a choisi la firme GRICS (à but non lucratif, propriété des commissions scolaires) et l'a chargée de/d' :

- Acquérir les droits sur un système (système choisi : SARRA).

- Superviser les améliorations ou modifications.

- Héberger le système et le rendre accessible à toutes les commissions scolaires et au ministère de l'Éducation.

- Assurer l'entretien et le service aux usagers.

- Permettre les liens avec les autres systèmes informatiques utilisés par les commissions scolaires.

7. Effectuer un banc d'essai.

8. Procéder aux modifications du système.

9. Signer les ententes de service entre les commissions scolaires et la GRICS. 
10. Implanter le système sur les serveurs de la GRICS.

11. Former les personnels des commissions scolaires et du ministère.

12. Visiter des écoles, faire des inventaires et audits d'état et saisir les renseignements.

13. Faire saisir les différents éléments par les commissions par étapes.

14. Évaluer le déficit d'entretien. Cette évaluation se fera en 2008, soit six ans après la dernière opération d'évaluation quantitative de l'état du parc immobilier du réseau primaire-secondaire public du Québec. (En 2001/02, le déficit d'entretien était de l'ordre de $8 \%$ de la valeur de la reconstruction du parc.)

\section{Principales caractéristiques recommandées d'un système de gestion des actifs immobiliers}

Suite aux travaux réalisés dans le cadre de ce projet et aux nombreuses discussions avec les gestionnaires immobiliers, voici des recommandations quant aux caractéristiques essentielles que devrait comprendre un système de gestion des actifs immobiliers.

\section{Inventaire des actifs}

Comme son nom l'indique, ce système doit inclure tous les renseignements complets sur les actifs d'un parc immobilier, conservés à l'intérieur d'une base de données facile à mettre à jour. De plus, la classification des actifs devrait être basée sur un système tel UNIFORMAT II, qui est la référence dans l'industrie. Un inventaire standardisé est la base uniformisée pour des analyses, pour établir des budgets de financement équitables et pour faciliter l'intégration avec d'autres systèmes.

\section{Évaluation de l'état des actifs}

Le système doit permettre d'enregistrer les données relatives à l'état des actifs immobiliers tel que des cotes d'état, la vie utile résiduelle, la courbe de détérioration, les défectuosités, les mesures correctives (coût et année estimée), des remarques, des documents, des images et la priorité des interventions.

\section{Déficit d'entretien accumulé}

Un SGA doit permettre de calculer le déficit d'entretien accumulé (coûts de réhabilitation ou de remplacement qui n'ont pu être effectués jusqu'à maintenant en raison des contraintes budgétaires ou autres) et de présenter les résultats par bâtiment et par catégorie d'actif (enveloppe du bâtiment, chauffage, ventilation et conditionnement d'air, éléments électriques, etc.). Le système doit contenir une base de données avec des coûts de remplacement préalablement établis pour les principaux composants des immeubles.

\section{Projection des coûts de maintien des actifs}

À l'aide des activités de réhabilitation, des prévisions des coûts de remplacement et de la durée de vie utile résiduelle saisis, le système doit pouvoir fournir des projections des coûts de maintien des actifs s'échelonnant sur une longue période, par exemple sur 25 ans. Le système doit permettre également de calculer le fonds d'allocation de réserve annuel en tenant compte des variables au niveau des taux d'inflation ou d'indexation annuelle des coûts de la construction.

\section{Priorités et planification des travaux}

Lorsque I'enveloppe budgétaire annuelle disponible pour la réalisation de travaux est établie, le SGA doit faciliter la préparation de la liste des travaux prioritaires à présenter pour approbation par les autorités, en faisant ressortir des indicateurs de performance tel que l'index de l'état des infrastructures (Facility Condition Index) de I'APPA (Association of Higher Education Facilities Officers, États-Unis) ou la catégorie de travaux correspondant à l'intervention (environnement, santé, vétusté, économies d'énergie). Une fois autorisée, la programmation est mise en œuvre et les renseignements sur les composants qui ont été remplacés ou réparés sont corrigés pour tenir compte de leur nouvel état.

\section{Calcul des coûts de maintenance préventive et de réparation}

En plus d'inclure une base de données des coûts de remplacement pour les principaux composants des immeubles, le SGA doit incorporer les coûts de réparation et maintenance qui permettront de répartir les budgets aux endroits présentant les plus grands besoins.

\section{Module générateur de rapports efficace}

L'un des éléments essentiels et fort utiles d'un SGA est d'incorporer un module qui permette de générer facilement des rapports de gestion, des figures ou des photographies, des bilans de l'état du parc, des listes de déficiences et de travaux par catégorie, des programmations des travaux urgents, et des rapports sur l'évolution du déficit d'entretien en fonction des budgets annuels consacrés au maintien des actifs. Le générateur de rapports doit pouvoir utiliser et exploiter toutes les données relatives aux actifs inventoriés, à leur état et à leurs coûts.

\section{Article de :}

Dino Gerbasi

Vice-Président Recherche et Développement

GES Technologies Inc.

Québec, Canada

Télécopie : 005142576090

dgerbasi@ges-int.com

et Gilles Marchand

Directeur de l'Équipement scolaire

Ministère de l'Éducation du Québec

Québec, Canada

Télécopie : 0014186439224

gilles.marchand@meq.gouv.qc.ca 